\title{
Computation of Even-Odd Harmonious Labeling of Certain Family of Acyclic Graphs
}

\author{
M. Kalaimathi, B. J. Balamurugan
}

Abstract: Let $G(V, E)$ be a graph with p number of vertices and $q$ number of edges. An injective function $\boldsymbol{f}: \boldsymbol{V} \rightarrow\{1,3,5, \ldots, 2 \boldsymbol{p}-1\}$ is called an even-odd harmonious labeling of the graph $G$ if there exists an induced edge function $\boldsymbol{f}^{*}: \boldsymbol{E} \rightarrow\{0,2, \ldots, 2(\boldsymbol{q}-1)\}$ such that

i) $f^{*}$ is bijective function

ii) $f^{*}(e=u v)=(f(u)+f(v))(\bmod 2 q)$

The graph obtained from this labeling is called even-odd harmonious graph.

Keywords : Graphs, Even-Odd Harmonious Labeling, Injective Function, Bijective Function.

\section{INTRODUCTION}

Labeling of a graph is an interesting and potential area of research in graph theory. It deals how the vertices and edges of the graph are labeled through well defined Mathematical functions [1]. The recent advancements and applications of various labelings of graphs have been updated by Gallian [5]. In the year 1967, Rosa in [9] introduced the graph labeling concepts. N. Lakshmi Prasana etl., in the paper [7] listed out the applications of graph labeling. We refer the text books written by Harary [4] and D. B.West [11] for the concepts and terminologies in graph theory.

The harmonious graph were introduced [3] in the year 1980. Further Z. Liang etl., [8] introduced the odd harmonious graphs in the year 2009 and P.B. Sarasija etl., [10] introduced the even harmonious graphs in the year 2011. Subsequently in the year 2015, Adalin Beatress and Sarasija in [2] introduced the even-odd harmonious graphs. Following this in the year 2019, we have proved that the graphs which are obtained through certain graph operations admit the even-odd harmonious labeling in the paper [6]. In this paper, we prove further the existence of even-odd harmonious labeling to certain family of acyclic graphs.

\section{Preliminaries}

In this section, we recall the definitions of certain graphs pertaining to this paper.

Revised Manuscript Received on July 22, 2019.

* Correspondence Author

M. Kalaimathi *, Mathematics Division, School of Advanced Sciences, VIT University, Chennai, India. Email: kalaimathi.m2018@ vitstudent.ac.in

B. J. Balamurugan, Mathematics Division, School of Advanced Sciences, VIT University, Chennai, India. Email: balamurugan.bj@vit.ac.in

\section{Definition 2.1}

A caterpillar is a graph obtained from a path graph by adding one or more pendant edges to the vertices of the path. The caterpillars can be drawn as a bipartite graph in a zigzag vertical fashion with one partioned set on the left side and the other partioned set on the right side. A caterpillar with $m$ vertices on the path and $t$ pendant edges is denoted $\mathrm{Cat}_{m}^{+t}(l, r)$, where the vertex set of the caterpillar can be partioned into two sets with $l$ and $r$ vertices.

\section{Definition 2.2}

1-regular lobster is a graph obtained from a path of $n$ vertices $P_{n}$ by attaching a path of length 2 to each vertex of $P_{n}$.

\section{Definition 2.3}

Let $P_{n}$ be a path with $n$ vertices. A coconut tree $C T_{n, m}$ is a graph obtained by joining $m$ new pendent edges at an end vertex of $P_{n}$.

\section{Definition 2.4}

A tree $T$ with $n$ legs and $l$ length is called spider tree if exactly one vertex of degree greater than or equal to 3 .

\section{Definition 2.5}

A complete bipartite graph $K_{1, n}$ is called a star graph $S_{n}$ and it has $n+1$ vertices and $n$ edges.

\section{EVEN-ODd HARMONIOUS LABELING OF GRAPHS}

In this section, we provide the notion of even-odd harmonious labeling of graphs [2].

\section{Definition 3.1}

Let $G(V, E)$ be a graph with $p$ number of vertices and $q$ number of edges. An injective function $f: V \rightarrow\{1,3,5, \ldots, 2 p-1\} \quad$ is called an even-odd harmonious labeling of the graph $G$ if there exists an induced edge function $f^{*}: E \rightarrow\{0,2, \ldots, 2(q-1)\}$ such that

i) $f^{*}$ is bijective function

ii) $f^{*}(e=u v)=(f(u)+f(v))(\bmod 2 q)$ 


\section{Computation of Even-Odd Harmonious Labeling of Certain Family of Acyclic Graphs}

\section{Definition 3.2}

A graph obtained by assigning numbers to the vertices and edges through an even-odd harmonious labeling is known as even-odd harmonious graph.

\section{Remark}

We use the phrase "EOH labeling of graph" instead of the phrase "even-odd harmonious labeling of graph" in this paper for simplicity.

\section{Example 3.1}

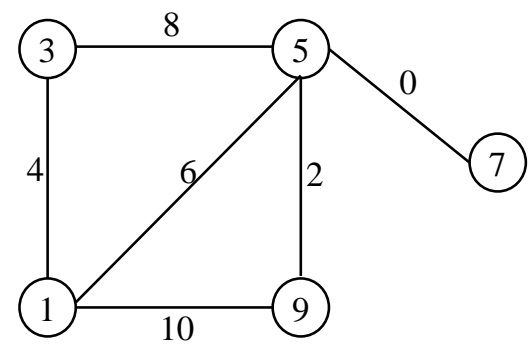

Fig. 1 EOH labeling of $G$

\section{Even-OdD HaRmonious Labeling OF ACYCLIC GRAPHS}

In this section, we prove certain family of acyclic graphs admit the EOH labeling.

\section{Theorem 4.1}

The caterpillar graph $c a t_{m}^{+t}(l, r)$ admits an EOH labeling when $m \geq 3, t \geq 1$.

\section{Proof}

Let $V=\left\{l_{i}: 1 \leq i \leq l\right\} \cup\left\{r_{j}: 1 \leq j \leq r\right\}$ be the vertex set of $\operatorname{cat}_{m}^{+t}(l, r)$ where the vertices of the left side are $l_{i}$ and the vertices of right side are $r_{i}$. Let $E=\left\{e_{i j}=l_{i} r_{j}: 1 \leq i \leq l, 1 \leq j \leq r\right\}$ be the edge set of the caterpillar graph $c a t_{m}^{+t}(l, r)$. Here the caterpillar graph has $p=m+t$ vertices and $q=m+t-1$ edges.

Define an injective function $f: V \rightarrow\{1,3, \ldots, 2(m+t)-1\}$ such that

$$
\begin{aligned}
& f\left(l_{i}\right)=2 i-1,1 \leq i \leq l \\
& f\left(r_{j}\right)=2 l+2 j-1,1 \leq j \leq r
\end{aligned}
$$

and an induced edge function $f^{*}: E \rightarrow\{0,2,4, \ldots, 2(m+t-1)-2\}$ such that

$$
\begin{array}{r}
f^{*}\left(e_{i j}\right)=f^{*}\left(l_{i} r_{j}\right)=(2 l+2 i)(\bmod 2 q), \\
1 \leq i \leq l, 1 \leq j \leq r
\end{array}
$$

where $f^{*}$ is bijective. The functions $f$ and $f^{*}$ give $\mathrm{EOH}$ labeling of $G$.
Therefore, the caterpillar $c a t_{m}^{+t}(l, r)$ is an EOH graph when $n \geq 1$.

\section{Example 4.1}

An EOH labeling of $c a t_{8}^{+5}(6,7)$ is shown in Fig. 2

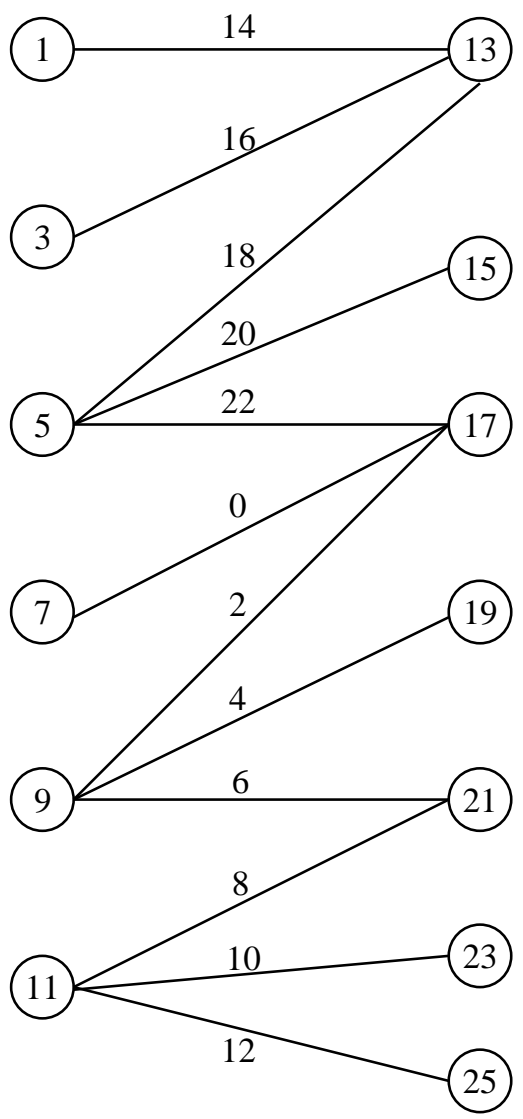

Fig. 2 EOH labeling of $c a t_{8}^{+5}(6,7)$

\section{Theorem 4.2}

The 1-regular lobster graph admits an EOH labeling when $n \geq 2$.

\section{Proof}

Let $V=\left\{u_{i}: 1 \leq i \leq n\right\} \cup\left\{u_{i j}: 1 \leq i \leq n\right.$ and $\left.j=1,2\right\}$ be the vertex set of 1-regular lobster where $u_{i}$ are the vertices of the path $P_{n}$ and $u_{i j}$ are the vertices, which are adjacent to $u_{i}$ for $1 \leq i \leq n$ and $j=1,2$.

Let

$$
E=\left\{e_{i}=u_{i} u_{i+1}: 1 \leq i \leq n-1\right\} \cup
$$

$\left\{e_{i j}=u_{i} u_{i j}: 1 \leq i \leq n\right.$ and $\left.j=1,2\right\}$ be the edge set of the 1 -regular lobster. Here the 1-regular lobster has $p=3 n$ vertices and $q=3 n-1$ edges.

Define an injective function $f: V \rightarrow\{1,3, \ldots, 2(3 n)-1\}$ such that 
Case (i) $n \equiv 0(\bmod 2)$

$$
\begin{aligned}
& f\left(u_{i}\right)=\left\{\begin{array}{r}
3(i-1)+1, \text { if } i \text { is odd } \\
3 n+4+3(i-2)+1, \text { if } i \text { is even }
\end{array}\right. \\
& f\left(u_{i 1}\right)=\left\{\begin{array}{c}
3(i-1)+3 n+1, \text { if } \text { i is odd } \\
3(i-2)+5, \text { if } \text { i is even }
\end{array}\right. \\
& f\left(u_{i 2}\right)=\left\{\begin{array}{c}
3(i-1)+3, \text { if } \text { i is odd } \\
3(n+i)-3, \text { if } \text { i is even }
\end{array}\right.
\end{aligned}
$$

Case (ii) $n \equiv 1(\bmod 2)$

$$
\begin{aligned}
& f\left(u_{i}\right)=\left\{\begin{array}{l}
3(i-1)+1, \text { if } i \text { is odd } \\
3(n+i), \text { if } \text { i is even }
\end{array}\right. \\
& f\left(u_{i 1}\right)=\left\{\begin{array}{l}
3(n+i)-1, \text { if } \text { i is odd } \\
3(i-2)+5, \text { if } \text { i is even }
\end{array}\right. \\
& f\left(u_{i 2}\right)=\left\{\begin{array}{c}
3(i-1)+3, \text { if } \text { i is odd } \\
3(n+i-1)+1, \text { if } \text { i is even }
\end{array}\right.
\end{aligned}
$$

and an induced edge

$f^{*}: E \rightarrow\{0,2,4, \ldots, 2(3 n-1)-2\}$ such that

Case (i) $n \equiv 0(\bmod 2)$

$f^{*}\left(e_{i}\right)=f^{*}\left(u_{i} u_{i+1}\right)=(3 n+4+6(i-1)+2)(\bmod 2 q)$, $1 \leq i \leq n-1$

$f^{*}\left(e_{i 1}\right)=f^{*}\left(u_{i} u_{i 1}\right)=\left\{\begin{array}{c}(3 n+6(i-1)+2)(\bmod 2 q), \text { if } i \text { is odd } \\ (3 n+6 i-2)(\bmod 2 q), \text { if } \text { i is even }\end{array}\right.$ $f^{*}\left(e_{i 2}\right)=f^{*}\left(u_{i} u_{i 2}\right)=\left\{\begin{array}{l}(3 n+6(i-1)+4)(\bmod 2 q), \text { if } \text { i is odd } \\ (3(n+2 i-2)+2)(\bmod 2 q), \text { if i is even }\end{array}\right.$

Case (ii) $n \equiv 1(\bmod 2)$

$$
\begin{aligned}
& f^{*}\left(e_{i}\right)=f^{*}\left(u_{i} u_{i+1}\right)=(3(n+2 i)+1)(\bmod 2 q), 1 \leq i \leq n-1 \\
& f^{*}\left(e_{i 1}\right)=f^{*}\left(u_{i} u_{i 1}\right)=\left\{\begin{array}{l}
(3(n+2 i-1)-1)(\bmod 2 q), \text { if } i \text { is odd } \\
(3(n+2 i+1)+2)(\bmod 2 q), \text { if } \text { i is even }
\end{array}\right. \\
& f^{*}\left(e_{i 2}\right)=f^{*}\left(u_{i} u_{i 2}\right)=\left\{\begin{array}{l}
(3 n+6(i-1)+5)(\bmod 2 q), \text { if } \text { i is odd } \\
(3(n+2 i-2)+3)(\bmod 2 q), \text { if } \text { i is even }
\end{array}\right.
\end{aligned}
$$

where $f^{*}$ is bijective. Here in all cases $f$ and $f^{*}$ defines the EOH labeling of $G$. Thus, the 1-regular lobster admits EOH labeling when $n \geq 2$.

\section{Example 4.2}

An EOH labeling of 1 -regular lobster is shown in Fig. 3

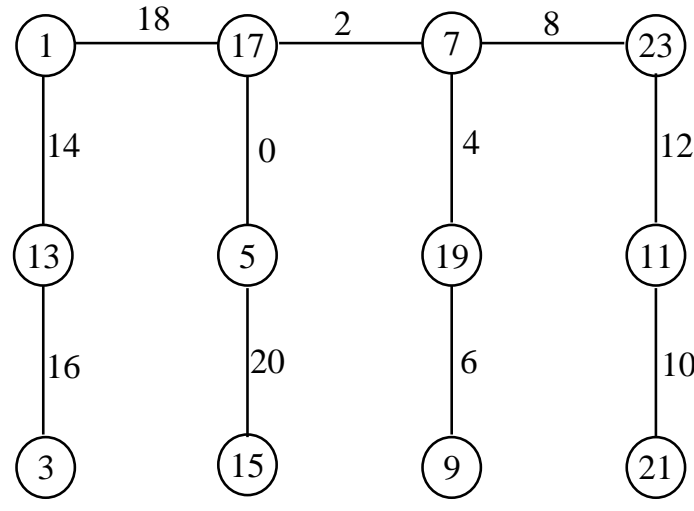

Fig. 3 EOH labeling of 1 -regular lobster

\section{Theorem 4.3}

The coconut tree graph $C T_{n, m}$ admits an $\mathrm{EOH}$ labeling when $n \geq 2, m \geq 2$.

\section{Proof}

Let $V=\left\{u_{i}: 1 \leq i \leq n\right\} \cup\left\{v_{j}: 1 \leq j \leq m\right\}$ be the vertex set of coconut tree where $u_{i}$ are the vertices of the path $P_{n}$ and $v_{j}$ are the $m$ new pendent vertices at an end vertex of the path $P_{n}$.

$$
\text { Let } E=\left\{e_{i}=u_{i} u_{i+1}: 1 \leq i \leq n\right\} \cup
$$

$\left\{e_{i j}=u_{i} v_{j}: i=n, 1 \leq j \leq m\right\}$ be the edge set of coconut tree. Here the coconut tree has $p=n+m$ vertices and $q=n+m-1$ edges.

Define an injective function $f: V \rightarrow\{1,3, \ldots, 2(n+m)-1\}$ such that

Case (i) $n \equiv 1(\bmod 2)$

$$
\begin{aligned}
& f\left(u_{i}\right)=\left\{\begin{array}{c}
i, \text { if } i \text { is odd } \\
n+i, \text { if i is even }
\end{array}\right. \\
& f\left(v_{j}\right)=2 n+2 j-1,1 \leq j \leq m
\end{aligned}
$$

Case (ii) $n \equiv 0(\bmod 2)$

$$
\begin{aligned}
& f\left(u_{2 i-1}\right)=n+i, 1 \leq i \leq \frac{n}{2} \\
& f\left(u_{2 i}\right)=2 i-1,1 \leq i \leq \frac{n}{2}
\end{aligned}
$$$$
f\left(v_{j}\right)=2 n+2 j-1,1 \leq j \leq m
$$

and an induced edge function $f^{*}: E \rightarrow\{0,2,4, \ldots 2(n+m-1)-2\}$ such that 


\section{Computation of Even-Odd Harmonious Labeling of Certain Family of Acyclic Graphs}

Case (i) $n \equiv 1(\bmod 2)$

$$
f^{*}\left(e_{i}\right)=f^{*}\left(u_{i} u_{i+1}\right)=(n+1+2 i)(\bmod 2 q), 1 \leq i \leq n-1
$$

$f^{*}\left(e_{i j}\right)=f^{*}\left(u_{i} v_{j}\right)=(2(m+n-1)+2 j)(\bmod 2 q), i=n, 1 \leq j \leq m$

Case (ii) $n \equiv 0(\bmod 2)$

$$
\begin{aligned}
& f^{*}\left(e_{i}\right)=f^{*}\left(u_{i} u_{i+1}\right)=(n+2 i)(\bmod 2 q), 1 \leq i \leq n \\
& f^{*}\left(e_{i j}\right)=f^{*}\left(u_{i} v_{j}\right)=(2 n+2 j+2)(\bmod 2 q), i=n, 1 \leq j \leq m
\end{aligned}
$$

where, $f^{*}$ is bijective. The functions $f$ and $f^{*}$ provides the numbers on vertices and edges satisfying the conditions of EOH labeling of $G$. Thus, the coconut tree $C T_{n, m}$ admits EOH labeling when $n \geq 2, m \geq 2$.

\section{Example 4.3}

An EOH labeling of $C T_{3,5}$ and $C T_{4,5}$ is shown in Fig. 4 and Fig. 5

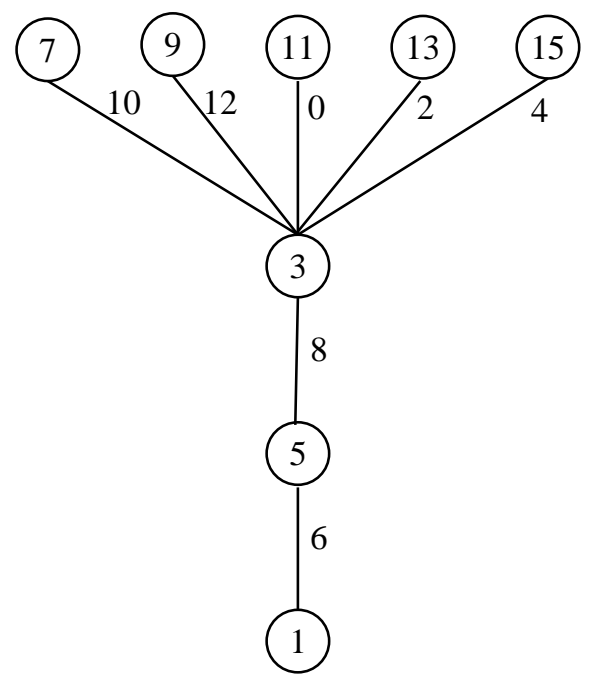

Fig. 4 EOH labeling of $C T_{3,5}$

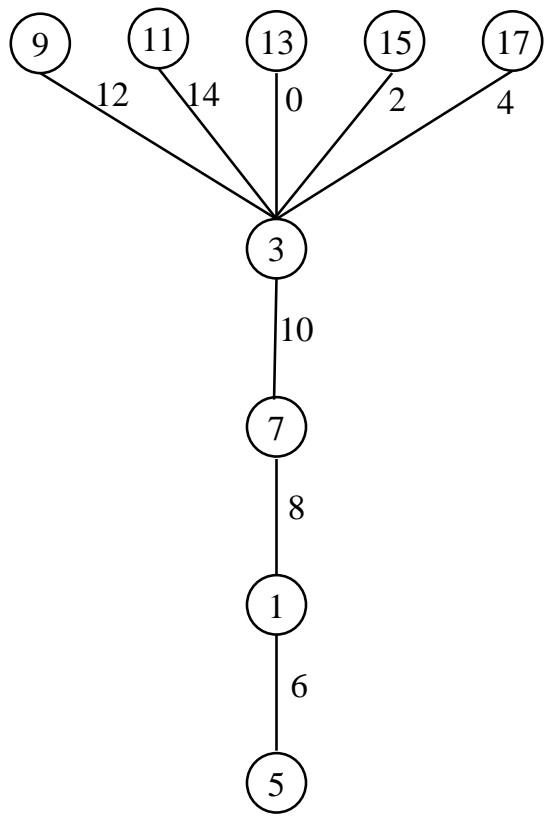

Fig. 5 EOH labeling of $C T_{4,5}$

\section{Theorem 4.4}

The spider tree graph with $n$ legs and $l$ length admits an EOH labeling when $n \geq 3, n \equiv 1(\bmod 2)$ and $l \equiv 0(\bmod 2)$.

\section{Proof}

Let $V=\{v\} \cup\left\{v_{i j}: 1 \leq i \leq n, 1 \leq j \leq l\right\}$ be the vertex set of spider tree where $v$ is a center vertex, $v_{i j}$ are the $n$ legs and $l$ length of the spider tree graph. Let $E=\left\{e_{i j}=v_{i j} v_{i j+1}: 1 \leq i \leq n\right.$ and $\left.1 \leq j \leq l-1\right\} \cup$

$\left\{e_{i}=v v_{i 1}: 1 \leq i \leq n\right\} \quad$ be the edge set of the spider tree. Here the spider tree has $p=n l+1$ vertices and $q=n l$ edges.

Define an injective function $f: V \rightarrow\{1,3, \ldots, 2(n l+1)-1\}$ such that

$$
f(v)=1
$$

$f\left(v_{i j}\right)=\left\{\begin{array}{l}l(n+i-1)+j+2, \text { if both } i \text { and } j \text { are odd } \\ l(n+i-1)+j+1, \text { if both } i \text { and } j \text { are even } \\ (i-1) l+j+1, \text { if } i \text { is odd and } j \text { is even } \\ (i-1) l+j+2, \text { if } i \text { is even and } j \text { is odd }\end{array}\right.$

and an induced edge function $f^{*}: E \rightarrow\{0,2,4, \ldots 2 n l-2\}$ such that

$$
\begin{aligned}
& f^{*}\left(e_{i j}\right)=f^{*}\left(v_{i j} v_{i j+1}\right)=(l(n+3 i-2)+2(j-1))(\bmod 2 q), \\
& 1 \leq i \leq n \text { and } 1 \leq j \leq l-1 \\
& f^{*}\left(e_{i}\right)=f^{*}\left(v v_{i 1}\right)=\left\{\begin{array}{c}
(l(n+i-1)+3)(\bmod 2 q), \text { if } i \text { is odd } \\
(l(i-1)+3)(\bmod 2 q), \text { if i is even }
\end{array}\right.
\end{aligned}
$$

where $f^{*}$ is bijective. Both the functions $f$ and $f^{*}$ provides numbers to the vertices and edges satisfying EOH labeling of $G$. Therefore, the spider tree with $n$ legs and $l$ length admits EOH labeling. 


\section{Example 4.4}

An EOH labeling of the spider tree with 5 legs and length 4 is shown in Fig. 6

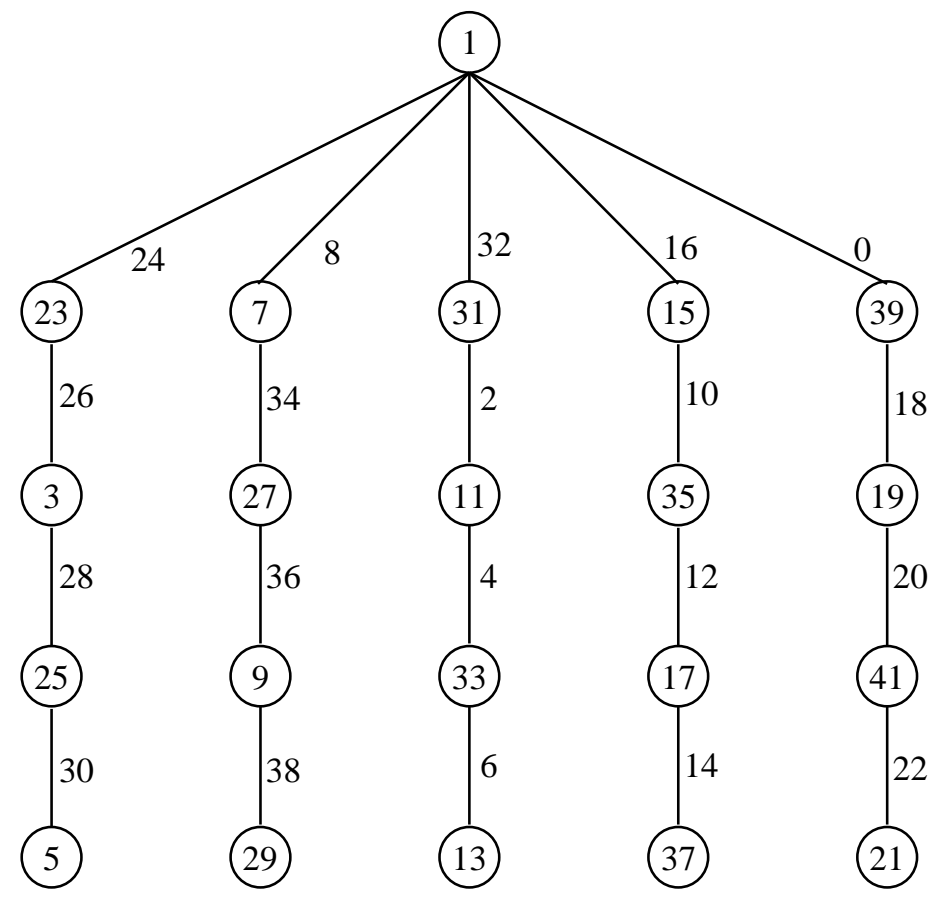

Fig. 6 EOH labeling of spider tree with 5 legs and length 4

\section{Theorem 4.5}

The star graph $S_{m, 3}$ admits an EOH labeling when $m \equiv 1(\bmod 2)$.

\section{Proof}

Let

$V=\{x\} \cup\left\{u_{i}: 1 \leq i \leq m\right\} \cup\left\{v_{i}: 1 \leq i \leq m\right\} \cup\left\{w_{i}: 1 \leq i \leq m\right\}$ be the vertex set of star graph where $x$ is a center vertex, $u_{i}, v_{i}, w_{i}$ are the vertices of the path $P_{3}$ for $1 \leq i \leq m$. Let $E=\left\{e_{i}=u u_{i}: 1 \leq i \leq m\right\} \cup\left\{g_{i}=u_{i} v_{i}: 1 \leq i \leq m\right\}$ $\cup\left\{h_{i}=v_{i} w_{i}: 1 \leq i \leq m\right\} \quad$ be the edge set of the star graph $S_{m, 3}$. Here the star graph has $p=3 m+1$ vertices and $q=3 m$ edges.

Define an injective function $f: V \rightarrow\{1,3, \ldots, 2(3 m+1)-1\}$ such that

$$
\begin{aligned}
& f(x)=2 m+1 \\
& f\left(u_{1}\right)=6 m+1 \\
& f\left(u_{2 i}\right)=4 i-1,1 \leq i \leq \frac{m}{2} \\
& f\left(u_{2 i+1}\right)=2 m+4 i+1,1 \leq i \leq \frac{m}{2} \\
& f\left(v_{2 i}\right)=2 m+4 i-1,1 \leq i \leq \frac{m}{2} \\
& f\left(v_{2 i-1}\right)=4(i-1)+1,1 \leq i \leq \frac{m}{2} \\
& f\left(w_{1}\right)=4 m+1
\end{aligned}
$$

$$
\begin{aligned}
& f\left(w_{2 i}\right)=5 m+2-2 i, 1 \leq i \leq \frac{m}{2} \\
& f\left(w_{2 i+1}\right)=6 m-2 i+1,1 \leq i \leq \frac{m}{2}
\end{aligned}
$$

and an induced edge function $f^{*}: E \rightarrow\{0,2,4, \ldots 2(3 m)-2\}$ such that

$$
\begin{aligned}
& f^{*}\left(e_{1}\right)=f^{*}\left(u u_{1}\right)=(2(m+1))(\bmod 2 q) \\
& f^{*}\left(e_{2 i}\right)=f^{*}\left(u u_{2 i}\right)=(2 m+4 i)(\bmod 2 q), 1 \leq i \leq \frac{m}{2} \\
& f^{*}\left(e_{2 i+1}\right)=f^{*}\left(u u_{2 i+1}\right)=(4 m+4 i+2)(\bmod 2 q), 1 \leq i \leq \frac{m}{2}
\end{aligned}
$$$$
f^{*}\left(g_{1}\right)=f^{*}\left(u_{1} v_{1}\right)=2
$$$$
f^{*}\left(g_{i+1}\right)=f^{*}\left(u_{i+1} v_{i+1}\right)=(2(m+1)+4 i)(\bmod 2 q), 1 \leq i \leq m
$$$$
f^{*}\left(h_{1}\right)=f^{*}\left(v_{1} w_{1}\right)=(4 m+2)(\bmod 2 q)
$$$$
f^{*}\left(h_{2 i}\right)=f^{*}\left(v_{2 i} w_{2 i}\right)=(m+2 i+1)(\bmod 2 q), 1 \leq i \leq \frac{m}{2}
$$$$
f^{*}\left(h_{2 i+1}\right)=f^{*}\left(v_{2 i+1} w_{2 i+1}\right)=(2+2 i)(\bmod 2 q), 1 \leq i \leq \frac{m}{2}
$$

where $f^{*}$ is bijective. The functions $f$ and $f^{*}$ give $\mathrm{EOH}$ labeling of $G$. Thus, the star $S_{m, 3}$ admits EOH labeling when $m \equiv 1(\bmod 2)$.

\section{Example 4.5}

An EOH labeling of $S_{5,3}$ is shown in Fig. 7

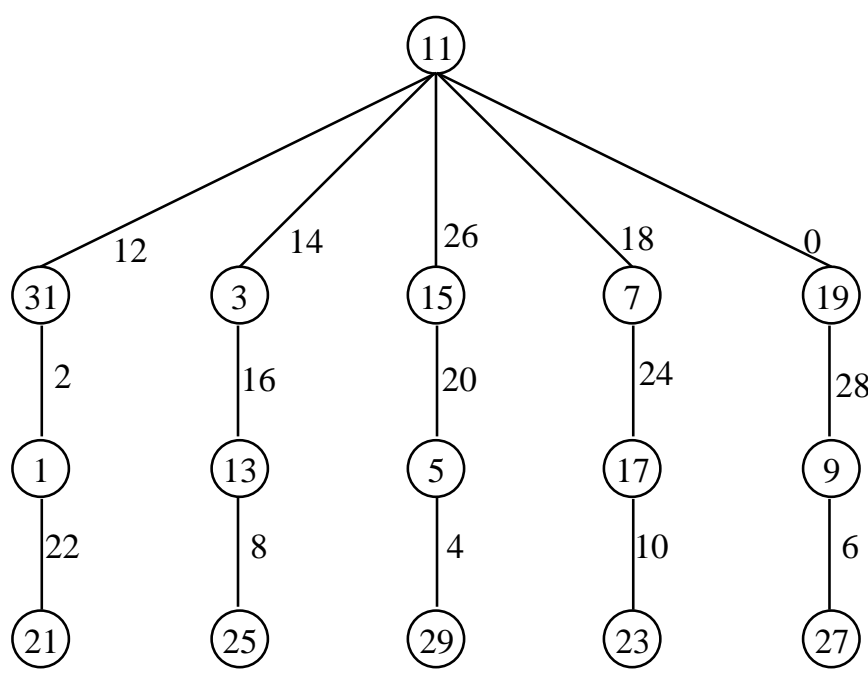




\section{Computation of Even-Odd Harmonious Labeling of Certain Family of Acyclic Graphs}

\section{CONCLUSION}

In this paper, we have proved that the family of acyclic graphs such as caterpillar, 1-regular lobster graph, coconut tree, spider tree and star graph admit the EOH labeling.

\section{FUTURE SCOPE}

We can find a family of cyclic graphs which will admit this EOH labeling.

\section{REFERENCES}

1. B.D. Acharya , S.M Hegde, Arithmetic Graphs. J. Graph Theory, Vol. 14, No.3, 275-299, (1990).

2. N. Adalin Beatress, P.B Sarasija, Even-Odd Harmonious Graphs, International Journal of Mathematics and Soft Computing, Vol. 5, No. 1, 23-29, (2015).

3. R.L Graham, N.J.A Sloane, On Additive Bases and Harmonious Graphs, SIAM Journal on Algebraic and Discrete Methods, Vol. 1, No. 4, 382-404, (1980).

4. F. Harary, Graph Theory, Addison-Wesley, Reading Mass, (1972).

5. Joseph A. Gallian, A Dynamic Survey of Graph Labeling, The Electronic Journal of Combinatorics, DS6, (2018).

6. M.Kalaimathi, B.J Balamurugan, Computation of Even-Odd Harmonious Labeling of Graphs Obtained by Graph Operations, Recent Trends in Pure and Applied Mathematics, AIP Conf. Proc. 2177, 020030-1 - 020030-9, (2019).

7. N. Lakshmi Prasana, K. Saravanthi, Nagalla Sudhakar, Applications of Graph Labeling in Major Areas of Computing Science. International Journal of Research in Computer and Communication Technology, Vol. 3, No. 8, (2014)

8. Z. Liang, Z. Bai Z, On The Odd Harmonious Graphs with Applications, J. Appl. Math. Comput., 29, 105-116, (2009).

9. A. Rosa, On Certin Valuations of the Vertices of a Graph, In Theory of Graphs(Internat.Sympos. Rome. 1966), Gordan and Breach, Newyork, Dunod, Paris, 349-359, (1967).

10. P. B. Sarasija, R. Binthiya, Even Harmonious Graphs with Applications, International Journal of Computer Science and Information Security, Vol. 9, No. 7, 161-163, (2011).

11. D. B. West, Introduction to Graph Theory, PHI Learning Private Limited, $2^{\text {nd }}$ Edition, (2009).

\section{AUTHORS PROFILE}

M.Kalaimathi, received her M.Sc. and M.Phil. degree in Mathematics from Madras Chiristian College, University of Madras, Chennai, India. She is currently pursuing Ph.D. in Mathematics at VIT University, Chennai Campus, Chennai, India. Her area of research is graph theory.

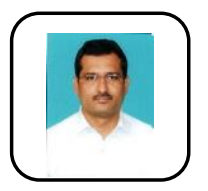

Dr. B.J.Balamurugan, received his $\mathrm{Ph} . \mathrm{D}$ degree in Mathematics from the University of Madras, Chennai, India. Currently, he is an Assistant Professor(Senior) of Mathematics in the School of Advanced Sciences at VIT University, Chennai Campus, Chennai, India. He has more than 22 years of teaching experience at Undergraduate and Postgraduate level courses. Dr.B.J.Balamurugan has published more than 32 research papers in various journals and conference proceedings. His research interest includes graph theory, graph grammars, fuzzy logic and Petri nets. 